\title{
Research on Application of Artificial Intelligence in Power System
}

\author{
Yujun Pan \\ Indiana University Bloomington, 107 S. Indiana Avenue Bloomington, \\ IN 47405-7000
}

\begin{abstract}
This paper briefly introduces the basic concept of artificial intelligence technology and points out its application in power system. The basic conceptions of artificial intelligence technology such as expert system, artificial neural network, fuzzy theory, genetic algorithm and so on are introduced briefly. From the practical point of view, the characteristics and problems of their application in power system fault diagnosis are analyzed. It is pointed out that the integrated use of a variety of artificial intelligence technology is the latest development trend of the application of artificial intelligence technology in power system, and put forward specific suggestions.
\end{abstract}

Keywords: application, artificial intelligence, power system

\section{Introduction}

Power system is composed of power generation equipment, transformers, transmission and distribution lines and electrical equipment, and many other components of the complex nonlinear dynamic system. With the continuous development of the power grid and the power to the market, people on the safe operation of power grid and power supply reliability requirements are getting higher and higher. Power system failure, requiring dispatchers to quickly and accurately identify the fault components and fault nature, timely treatment of failures and restore the normal operation of the power system. Power transmission and distribution system is the power system in the power system and power users to transfer energy and distribution of energy between the 
intermediate links, including the voltage level of the transmission and distribution lines and substations. His fault is unavoidable, and the scale of the power system is expanding and the application of various monitoring equipment makes the diagnosis of power transmission and distribution network is particularly important. Because the reliability index is an important factor affecting the reliability of the whole power system, the improvement of its reliability will bring great benefits to the safety, reliability and economic operation of the whole power system. Therefore, researchers have been committed to the development of advanced, accurate and efficient automatic fault diagnosis system.

The fault diagnosis of transmission and distribution network mainly analyzes the characteristics of alarm information, circuit breaker state change information and voltage and current measurement, etc., according to the logic of protection action and the experience of operating personnel. Because this process is difficult to use traditional mathematical methods to describe, and artificial intelligence technology because of its good at simulating the process of dealing with human problems, easy to take into account the experience and have a certain learning ability in this area has been a wide range of application. By analyzing the cognitive process of network defect judgment and applying the latest achievements of artificial intelligence technology such as comprehensive knowledge diagnosis, fuzzy theory and neural network, a comprehensive automatic logic analysis and judgment system is developed, which can analyze defects and provide supervision and treatment opinions, so that maintenance personnel to understand the issue more comprehensive, effective and targeted. Artificial intelligence technology is widely used in solving nonlinear problems, compared with the traditional method has an irreplaceable advantage. At present, a variety of artificial intelligence tools have been developed, such as expert system (ES), artificial neural network (ANN), fuzzy theory (FZ), heuristic search (HS) and genetic algorithm (GA).

\section{The application direction of artificial intelligence in the power system}

With the rapid development of China's power grid and the construction of "Sanhua" UHV power grid, more and more factors affect the safe operation of the power grid, the operation mechanism of the power grid is more and more complicated. In order to ensure the safe operation of the power grid, intelligent technology research also appears more important. In order to study the characteristics of the power system suitable for solving the problem through artificial intelligence tools, the problem is divided into two parts: real-time control and management planning.

Real-time control includes discrete and continuous control systems, in real time control is relatively simple, but the interaction between them makes the power system control is very complex. The system-wide data is transferred to the EMS via supervisory control and data acquisition (SCADA), and the control 
signals are sent from the EMS to the components. The whole process needs to be done synchronously, which requires the EMS to have real-time processing capability for a large amount of information. Monitoring and diagnosis is an important function of EMS. Artificial intelligence plays an important role in the field of condition monitoring and fault diagnosis. A variety of diagnosis strategies based on expert system and neural network have been developed at home and abroad.

\section{The main application methods}

\subsection{Application of Expert System (ES)}

An expert system is a computer program with expert experience and knowledge in a field that can be used as a human expert to make decisions through reasoning. A typical expert system consists of four parts: knowledge base, inference engine, knowledge acquisition mechanism and man-machine interface. The expert system has become the most mature artificial intelligence technology in the power system. A variety of expert systems have been developed at home and abroad for different areas of power systems: monitoring and diagnostics, grid dispatching, predictive accident screening, and system recovery. In particular, monitoring and fault diagnosis has become the most important applications of ES in power systems. According to the different ways of storing knowledge, the expert system can be divided into different forms, namely expert system based on shallow knowledge (experience knowledge), rules, decision tree, model, and objectoriented expert system. The model-based knowledge representation is suitable for real-time processing and is faster, simpler and easier to maintain than other methods such as rule-based (hypothetical) or heuristic reasoning. The typical application of expert system in fault diagnosis of transmission network is based on the rule of production rule, that is, the action logic of the protection circuit breaker and the diagnosis experience of operating personnel are expressed by rules, and the knowledge base of fault diagnosis expert system is formed, Alarm information to the knowledge base reasoning, fault diagnosis to obtain the conclusion. The fault diagnosis expert system based on production rule can be widely used by fault diagnosis and expert system based on production rule. Action logic for protection in the transmission network The relationship between the primary protection and the circuit breaker is easily expressed in an intuitive, modularized way; the expert system based on production rules allows for the addition, deletion or modification of rules to ensure that the diagnostic system Real-time and effectiveness; to a certain extent, solve the problem of uncertainty; be able to give the conclusions in line with human language habits and have the appropriate interpretation and so on. In addition, the framework expert system is good at expressing knowledge with classification structure, can express the correlation between things clearly, and can simplify the representation and storage of inheritance knowledge. There are also a few applications in the transmission network alarm information processing and fault diagnosis. 
Although the expert system can effectively simulate the process of fault diagnosis by fault diagnosis experts, there are still some shortcomings in the practical application. The main problems are the bottleneck of knowledge acquisition, the difficulty in maintaining knowledge, and the failure to solve the fault diagnosis effectively. Determine the factors that greatly affect the accuracy of fault diagnosis.

\subsection{Application of Artificial Neural Network (ANN)}

Artificial neural network (ANN) is an information processing system which simulates the structure of human brain and the process of human cognition. Since it was first put forward in 1943, artificial neural network (ANN) has rapidly developed into another important branch of artificial intelligence technology parallel to expert system. He has received more and more attention in the field of intelligent fault diagnosis, such as parallel distributed processing, adaptive and associative memory, and has shown great potential and opened a new research field for intelligent fault diagnosis. Application of ANN technology to achieve fault diagnosis is different from ES diagnostic methods. The ANN method adaptively adjusts the connection weights and thresholds in the ANN through a large number of standard learning and training, and implicitly distributes the acquired knowledge on the whole network and implements the pattern memory of the ANN. Therefore, ANN has powerful ability of knowledge acquisition, and can effectively deal with noise-containing data, to make up for the lack of ES method. Each artificial neural network is used for fault diagnosis. Each ANN is responsible for the diagnosis of a part of the system. Because of the rapid parallel processing ability and good classification ability of neural network, it is widely used in real-time control, monitoring and diagnosis of power system, short-term and long-term load forecasting, state evaluation and so on, and neural network based load forecasting technology Become one of the most successful applications of artificial intelligence in power system.

\subsection{Fuzzy Sets Theory (Fuzzy Sets Theory)}

In fault diagnosis, the relationship between faults and symptoms is often ambiguous, the ambiguity that comes from the fault and the uncertainty of the relationship between symptoms, but also from fault and symptomatic description of the imprecision on the concept, and thus diagnosis The fuzzy relation matrix is established between the fault symptom space and the fault cause space, which is based on the expert's experience in the traditional method to solve the fuzzy diagnosis problem. Commonly used method is to combine the fuzzy relation matrices generated by each fuzzy inference rule, or with or. With the development and improvement of fuzzy theory, some of the advantages of fuzzy theory are gradually paid attention to, such as fuzzy theory can be adapted to the uncertainty problem; the fuzzy knowledge base using language variables to express the experience of experts, closer to human expression; A plurality of possible solutions to the problem can be obtained and the degree of priority can be sorted according to the ambiguity of these schemes and the like. At present, 
fuzzy theory has been introduced into the field of fault diagnosis for transmission and distribution networks. Uncertainties in fault diagnosis of transmission networks can easily lead to erroneous results for expert systems that require strict matching searches. When the fuzzy theory is integrated into the expert system, the precise reasoning becomes the approximate reasoning, which enhances the fault tolerance of the expert system to a considerable extent.

In recent years, the application of fuzzy set theory in power system has made rapid progress: the application of multi-objective fuzzy decision-making method for fault location and fault type identification; given fuzzy set theory of distribution system power flow and state estimation method; And inference of the load level of the distribution system, the induction of various types of users with different factors change; with fuzzy set method of transformer protection principle, the difference between internal failure, inrush current and current transformer saturation external fault; seek to maintain power system security Run and make full use of the transmission capacity between the compromise solution; used in the distribution system loss fuzzy computing model, improve the calculation accuracy and so on.

\subsection{Genetic Algorithms (GA)}

Genetic algorithm is based on natural selection and genetic mechanism, in the computer simulation of biological evolutionary mechanism of the optimal search algorithm. He can search in complex and large search space adaptively and find the optimal or quasi-optimal solution, and the algorithm is simple, applicable and robust. Genetic algorithm has few restrictions on the solution of the problem and does not involve the complex mathematical process of solving the conventional optimization problem, and can get the global optimal solution or local optimal solution set, which is better than the traditional optimization technology.

Genetic algorithm can solve the problem of fault diagnosis from the point of view of optimization, especially in the case of complex fault or existence of protection, circuit breaker malfunction, it can give the global optimal or local optimal multiple possible diagnostic results. But how to establish a reasonable fault diagnosis model of transmission network is the main bottleneck of using genetic algorithm. If a reasonable mathematical model can be established, not only the genetic algorithm can be used to solve the fault diagnosis, but also other similar heuristic optimization algorithms can be used to solve the fault diagnosis problems, such as ant system algorithm and TABU search algorithm. From the comparison of different crossover and mutation algorithms, it can be seen that different crossover and mutation operators have great influence on the results, and how to determine the optimal crossover and mutation operators and their corresponding parameters need to be further studied.

\section{Conclusion}

In short, compare the existing artificial intelligence technology and explore new diagnostic techniques and theoretical methods, research and development of 
sophisticated intelligent transmission and distribution network fault diagnosis system are placed in front of research workers and it is the main direction of future development.

\section{References}

[1] Cai Zixing, Xu Guangyou. Artificial intelligence and its application. Beijing: Tsinghua University Press, pp.68-70, 2011

[2] Ni Yuanping. Research on the Method of Artificial Intelligence for Fault Diagnosis. Electric Drive Automation, 8 (5), pp.87- 91, 2013

[3] Ge Chao-qiang, Tang Guo-qing, Wang Lei. Experimental Intelligent Expert System for Fault Recovery. Power System Automation, 12(10), pp. 18- 20, 2012

[4] Tang Hua-jin, Chen Han-ping. Application of Artificial Intelligence Technology (AI) in Power System. Electric Power Construction, 9(6), pp.58- 61, 2011

[5] Han Zhen-xiang, Wen Fu-shuan, Zhang Qi. Application of artificial intelligence in electric power system. Power System Automation, 8(4), pp.85-87, 2014 